\title{
A NOTE ON REGULAR AND COMPLETELY REGULAR TOPOLOGICAL SPACES
}

\author{
MICHAEL J. NORRIS
}

For the purposes of this note we shall consider a topological space to be a $T$-space; that is, the family of open sets for the space is closed under finite intersection and arbitrary union and contains both the void set and the space itself.

We shall be interested in classes of topological spaces having the same points; this common set of points we call the basic point set for the spaces. It is well known that the class of topological spaces $\delta$ for a given basic point set can be partially ordered by means of the inclusion relation for the families of open sets. One such topological space is said to be greater than another if the family of open sets for the former includes the family of open sets for the latter.

With reference to this order relation, $S$ is a complete lattice. Indeed, if $\left\{S_{\lambda} \mid \lambda \in \Lambda\right\}$ is a subset of $S$ and $U_{\lambda}$ is the family of open sets for $S_{\lambda}$, then $\prod_{\lambda \in \Delta} V_{\lambda}$ is the family of open sets for the greatest lower bound of $\left\{S_{\lambda} \mid \lambda \in \Lambda\right\}$, and the family $U$ of open sets for the least upper bound of this subset is given by:

$$
\mathcal{U}=\left\{U \mid \exists \lambda_{\alpha 1}, \lambda_{\alpha 2}, \ldots, \lambda_{\alpha n_{\alpha}} ; U_{\lambda_{\alpha i}} \in U_{\lambda_{\alpha i}} ; U=\sum_{\alpha} U_{\lambda_{\alpha 1}} \cdots U_{\lambda_{\alpha n_{\alpha}}}\right\}
$$

Edwin Hewitt ${ }^{1}$ has shown that the least upper bound of a class of regular (completely regular) topological spaces with the same basic point set is itself a regular (completely regular) space provided the original class of spaces is completely ordered. We propose to remove the restriction of complete ordering.

TheOREM. If $S,\left\{S_{\lambda} \mid \lambda \in \Lambda\right\}$, is a class of regular (completely regular) topological spaces with the same basic point set, then $S$, the least upper bound of $S$, is regular (completely regular).

Proof. (a) Each member of $S$ is regular.

Let $s$ be any point of $S$, and let $U$ be an open subset of $S$ containing $s$. Then there exist $U_{\lambda_{i}}(i=1,2, \cdots, n)$ such that $U_{\lambda_{i}}$ is an open subset of $S_{\lambda_{i}}$ and

Presented to the Society, April 26, 1947; received by the editors September 26 1949.

${ }^{1}$ E. Hewitt, A problem of set-theoretic topology, Duke Math. J. vol. 10 (1943) pp 309-333. 


$$
s \in \prod_{i=1}^{n} U_{\lambda_{i}} \subset U
$$

Since $S_{\lambda_{i}}$ is regular, there exists an open subset of $S_{\lambda_{i}}, V_{\lambda_{i}}$, such that $s \in V_{\lambda_{i}}$ and $V_{\lambda_{i}}^{C_{\lambda_{i}}} \subset U_{\lambda_{i}}$ (where $V_{\lambda_{i}}^{C_{\lambda_{i}}}$ denotes the closure of $V_{\lambda_{i}}$ in $S_{\lambda_{i}}$ ). Let $V$ be $\prod_{i=1}^{n} V_{\lambda_{i}}$. Then $s \in V$ and $V$ is an open subset of $S$. Finally,

$$
V^{c}=\left(\prod_{i=1}^{n} V_{\lambda_{i}}\right)^{c} \subset \prod_{i=1}^{n} V_{\lambda_{i}}^{c} \subset \prod_{i=1}^{n} V_{\lambda_{i}}^{c \lambda_{i}} \subset \prod_{i=1}^{n} U_{\lambda_{i}} \subset U .
$$

Hence $S$ is a regular space.

(b) Each member of $S$ is completely regular.

Let $s_{0}$ be any point of $S$ and let $U$ be an open subset of $S$ containing $s_{0}$. We pick $U_{\lambda_{i}}(i=1,2, \cdots, n)$ as in (a). Then there exist $f_{i}(i=1,2, \cdots, n), f_{i}$ a continuous real-valued function on $S_{\lambda_{i}}$, such that

$$
\begin{aligned}
f_{i}\left(s_{0}\right) & =0, & & \\
0 \leqq f_{i}(s) & \leqq 1 & & \left(s \in S_{\lambda_{i}}\right), \\
f_{i}(s) & =1 & & \left(s \in U_{\lambda_{i}}^{\prime}\right) .
\end{aligned}
$$

Let $f$ be the least upper bound of $\left\{f_{i} \mid i=1,2, \cdots, n\right\}$. Then

$$
\begin{aligned}
f\left(s_{0}\right) & =0, & & \\
0 \leqq f(s) & \leqq 1 & & (s \in S), \\
f(s) & =1 & & \left(s \in U^{\prime}\right) .
\end{aligned}
$$

Since $S$ is stronger than $S_{\lambda_{i}}, f_{i}$ is continuous on $S$. Hence $f$ is continuous on $S$. Hence $S$ is completely regular.

A topological space having only the void set and the space itself as open sets is plainly regular (completely regular). Thus any family of topological spaces has a regular (completely regular) lower bound. Considering now the family of regular (completely regular) topological spaces for a given point set as a partially ordered set, we have a partially ordered set with a least element and with least upper bounds for all subsets. Such partially ordered sets are complete lattices. Of course, the greatest lower bound of a family of regular (completely regular) topological spaces in the new lattice need not be the same as the greatest lower bound of the family in the lattice of all topological spaces.

College of St. Thomas 